\title{
РЕЗУЛЬТАТЫ ЭНДОСКОПИЧЕСКОЙ ТРАНССФЕНОИДАЛЬНОЙ АДЕНОМЭКТОМИИ У ДЕТЕЙ С БОЛЕЗНЬЮ ИЦЕНКО-КУШИНГА
}

\author{
Иващенко О.В., Григорьев А.Ю., Азизян В.Н., Надеждина Е.Ю., Стребкова Н.А., Карева М.А., Никанкина Л.В., \\ Абросимов А.Ю.
}

ФГБУ «НМИЦ эндокринологии» Минздрава России, Москва

ЦЕЛЬ: оценить эффективность нейрохирургического лечения пациентов с болезнью Иценко-Кушинга (БИК) детского возраста.

МАТЕРИАЛЫ И МЕТОДЫ: в Исследование были включены пациенты с лабораторно подтвержденным центральным АКТГ-зависимым гиперкортицизмом, которым на момент проведения ТСА не исполнилось 18-ти лет, а также не проводилось какого-либо патогенетического лечения. Период исследования длился с 2008 по 2020 гг. В исследование вошло 32 пациента: 18 девочек (56\%) и 14 мальчиков (44\%). По данным предоперационной МРТ гипофиза диффузная неоднородность аденогипофиза была выявлена у 12 (38\%) пациентов, микроаденома у 17 (53\%), макроаденома - у 3 (9\%) соответственно. Гипофизарный генез АКТГ-зависимого гиперкортицизма подтвержден данными селективного забора крови из нижних каменистых синусов у 15 (47\%) пациентов: всем пациентам с неоднородностью аденогипофиза и 3 пациентам с микроаденомой, менее 6 мм. Селективная ТСА была проведена в 41\% (13 из 32) наблюдений. Различные виды расширенной, вплоть до гипофизэктомии в 59\% (19 из 32). Все 32 пациентам было выполнено рутинное морфологическое исследование удаленного биоптата. Катамнестические данные были собраны у 27 пациентов. Период катамнестического наблюдения составил 4-139 мес. Дополнительные методы лечения были проведены 13 (41\%) пациентам, из них 12 была выполнена повторная ТСА, 1 - повторная ТСА+радиохирургия.

PЕЗУЛЬТАТЫ: послеоперационная ремиссия после первой ТСА была достигнута в 22 наблюдениях (69\%), из них в 95\% (21 набл.) наступила надпочечниковая недостаточность. Персистенция гиперкортицизма сохранялась у 10 (31\%) пациентов. Послеоперационные осложнения после первичного нейрохирургического вмешательства были представлены: в 3 наблюдениях развитием гипотиреоза (9\%), в 12 несахарным диабетом (37\%). В единичных наблюдениях было отмечено развитие гипогонадизма (1 набл. (3\%)), депрессивный синдром (1 набл. (3\%)) и гипонатриемия (1 набл. (3\%)). Рецидив гиперкортицизма развился у 3 (9\%) пациентов. Ремиссия после дополнительных методов лечения была отмечена у $100 \%$ пациентов. 